\title{
Atendimento ao paciente politraumatizado na perspectiva do enfermeiro socorrista
}

\author{
Care to the polytraumatized patient from the perspective of a rescue nurse \\ Atención al paciente politraumatizado em perspectiva de la enfermera en primeros auxílios
}

Recebido: 15/02/2021 | Revisado: 22/02/2021 | Aceito: 23/02/2021 | Publicado: 03/03/2021

\author{
Raquel SilvaVon Ameln \\ ORCID: https://orcid.org/0000-0003-1482-0395 \\ Hospital Santo Antônio, Brasil \\ E-mail: raquelvonameln@gmail.com \\ Norlai Alves Azevedo \\ ORCID: https://orcid.org/0000-0002-5281-1329 \\ Universidade Federal de Pelotas, Brasil \\ E-mail: norlai2011@ hotmail.com \\ Josiele de Lima Neves \\ ORCID: https://orcid.org/0000-0002-8754-059X \\ Universidade Federal de Pelotas, Brasil \\ E-mail: josiele_neves@hotmail.com \\ Débora Eduarda Duarte do Amaral \\ ORCID: https://orcid.org/0000-0002-3371-9308 \\ Universidade Federal de Pelotas, Brasil \\ E-mail: deboraamaralp@gmail.com \\ Andressa Azambuja Pinto \\ ORCID: https://orcid.org/0000-0002-0937-571X \\ Hospital Santo Antônio, Brasil \\ E-mail: andressa_a_p@hotmail.com
}

\begin{abstract}
Resumo
Objetivos: Descrever a percepção dos enfermeiros sobre o atendimento ao paciente politraumatizado em um Pronto Socorro (PS). Metodologia: Estudo qualitativo, descritivo e exploratório. Os dados foram coletados por meio de entrevistas semiestruturada e analisados conforme análise temática. Resultados: Participaram sete enfermeiros assistenciais de um Pronto Socorro localizado no sul do Rio Grande do Sul. Os enfermeiros percebem a atuação de liderança como um diferencial desta categoria, com iniciativa durante a assistência e capacidade de coordenar ações gerenciais e assistenciais. A ausência de protocolos foi mencionada, porém, destacam que realizam capacitação e curso de aperfeiçoamento para suprir esta carência. Todos os enfermeiros referem a pouca infraestrutura como um fator negativo no atendimento. Em relação as facilidades no atendimento, destacou-se a necessidade de desempenhar atividades em equipe. Considerações finais: Destaca-se a importância de investir no aperfeiçoamento destes profissionais. A infraestrutura precária, com espaços reduzidos, ficou em evidência como uma dificuldade para realizar o atendimento.
\end{abstract}

Palavras-chave: Serviço hospitalar de emergência; Traumatismo múltiplo; Enfermagem em emergência.

\begin{abstract}
Objectives: To describe the nurses' perception of the care provided to polytrauma patients in an Emergency Room (ER). Methodology: Qualitative, descriptive and exploratory study. The data were collected through semi-structured interviews and analyzed according to thematic analysis. Results: Seven nurses from an Emergency Room located in the south of Rio Grande do Sul participated in this study. The nurses perceive leadership as a differential in their category, with initiative during assistance and the ability to coordinate management and assistance actions. The absence of protocols was mentioned, however, they highlight that they carry out training and improvement courses to fill this lack. All nurses refer to poor infrastructure as a negative factor in care. In relation to easinesses in care, the performance of team activities stood out. Final considerations: The importance of investing in the improvement of these professionals is highlighted. The precarious infrastructure, with reduced spaces, became evident as a difficulty to perform the health care.
\end{abstract}

Keywords: Emergency service hospital; Multiple trauma; Emergency nursing.

\section{Resumen}

Objetivos: Describir la percepción de las enfermeras sobre la atención de pacientes con traumatismos múltiples en una sala de emergencias. Metodología: estudio cualitativo, descriptivo y exploratorio. Los datos se recopilaron a través de entrevistas semiestructuradas y se analizaron de acuerdo con el análisis temático. Resultados: participaron siete enfermeras asistentes de una sala de emergencias ubicada en el sur de Rio Grande do Sul. Las enfermeras perciben el 
liderazgo como un diferencial en esta categoría, con iniciativa durante la asistencia y la capacidad de coordinar acciones de gestión y asistencia. Se mencionó la ausencia de protocolos, sin embargo, enfatizan que llevan a cabo cursos de capacitación y mejora para llenar esta falta. Todas las enfermeras se refieren a la infraestructura deficiente como un factor negativo en la atención. En cuanto a las instalaciones de servicio, se destacó la necesidad de realizar actividades en equipo. Consideraciones finales: se destaca la importancia de invertir en la mejora de estos profesionales. La precaria infraestructura, con espacios reducidos, se hizo evidente como una dificultad para realizar el servicio.

Palabras clave: Servicio de urgencia en hospital; Traumatismo múltiple; Enfermería de urgencia.

\section{Introdução}

As emergências recebem pacientes com diversos tipos de acidentes, as lesões são distintas e devem ser atendidas por profissionais qualificados. No Brasil, o Plano de Ação da Rede de Urgência e Emergência (RUE) visa possibilitar maior acessibilidade ao usuário e assegura um atendimento com mão de obra capacitada (Sontag, 2014). O politraumatismo faz parte desses índices, sendo considerado, a causa primordial de morte em adultos jovens, com maior predomínio para o sexo masculino (Praça, Matos, Magro, Fioravanti, \& Hermann, 2017).

O trauma pode ser considerado por lesões que causem alterações físicas e/ou funcionais, de natureza física, química ou acidental, já o politraumatismo é quando um indivíduo é acometido por múltiplas lesões, em um episódio, no qual é desencadeado uma troca de energia entre os tecidos e o meio (Carvalho, \& Saraiva, 2015). As lesões ou agravos ao estado de saúde são oriundos de um forte impacto, em consequência de um acidente ou ato de violência, intencional ou não (Corrêa, Silva, \& Santiago, 2018).

Neste contexto, o enfermeiro tem participação ativa no atendimento ao politraumatizado, atuando na supervisão, coordenação e avaliação das ações de enfermagem, além de minimizar as sequelas. Mesmo em espaços reduzidos, com precariedade de materiais, o enfermeiro deve estar capacitado para tomar decisões imediatas e preparado para realizar a assistência aos pacientes de maior complexidade. Para tanto, além das atribuições para prestar assistência de enfermagem a este perfil, o enfermeiro deve valorizar as ações educativas, participação em revisão de protocolos de atendimento e trabalhar junto a equipe multiprofissional (Santos, Lima, Pestana, Colomé, \& Erdmann, 2016).

Os treinamentos às equipes de atendimento ao trauma devem ser valorizados pelas instituições, principalmente aos profissionais da enfermagem que vivenciam jornadas de trabalho exaustivas e possuem baixa remuneração para arcar com cursos de qualificação. Pois, esta jornada excessiva de trabalho afeta a qualidade de vida, ocasiona sentimento de frustração e, pode trazer prejuízos ao paciente (Zappas, Costa, \& Pissaia, 2018).

Considerando a importância da atuação do enfermeiro como profissional proativo, qualificado e capaz de intervir positivamente em situações de elevada complexidade, justifica-se a relevância deste estudo por destacar a percepção de enfermeiros atuantes em um serviço de emergência, o que poderá subsidiar discussões sobre o atendimento ao politraumatizado, bem como contribuir para a abrangência desta temática para qualificar os serviços de urgência e emergência.

Com base no exposto, objetiva-se descrever a percepção dos enfermeiros sobre o atendimento ao paciente politraumatizado em um Pronto Socorro (PS).

\section{Metodologia}

Trata-se de um estudo qualitativo, descritivo e exploratório, realizado em um Pronto Socorro (PS) referência para 22 municípios da região sul do estado do Rio Grande do Sul. Este PS dispõe de médicos clínicos, cirurgiões (tórax, vascular), pediatras, neurocirurgiões e traumatologistas; a equipe de enfermagem conta com enfermeiros, auxiliares e técnicos de enfermagem.

O método qualitativo permite que a coleta de dados ocorra por meio de entrevistas com questões abertas, neste tipo de 
estudo a interpretação dos dados por parte do pesquisador é importante, estando vinculadas a sua opinião sobre o fenômeno em estudo (Pereira, Shitsuka, Parreira, \& Shitsuka, 2018).

O local do estudo dispõe de 12 enfermeiros assistenciais. Os participantes deste estudo foram sete enfermeiros, que obedeceram aos seguintes critérios de inclusão: estar realizando assistência de enfermagem por no mínimo um ano na unidade, permitirem o uso de gravador durante a entrevista e a publicação dos resultados, não estar em licença ou gozando de férias no período da coleta do estudo.

A coleta de dados foi realizada no período de abril a maio de 2017, nos turnos da manhã, tarde e noite, conforme o horário de trabalho do respectivo enfermeiro. O convite para participação, se deu de modo aleatório, e o início ocorreu conforme a disponibilidade do profissional, com duração média de 20 minutos. Para a produção de dados optou-se pela entrevista semiestruturada, com seis questões referentes aos dados sociodemográficos e quatro questões abertas envolvendo os temas de atuação do enfermeiro ao paciente politraumatizado e a utilização de protocolos de atendimento. As entrevistas foram realizadas individualmente em local privado, no próprio Pronto Socorro, mantendo-se a privacidade dos participantes.

A análise de dados foi realizada por meio da proposta operacional de Minayo, que se desdobra em três etapas: Préanálise, Exploração do material e Tratamento e interpretação dos resultados (Minayo, 2010). As entrevistas foram gravadas e o material proveniente das gravações foi fielmente transcrito pela pesquisadora, sendo após analisado e realizada uma discussão com a literatura disponível em relação ao tema.

As entrevistas iniciaram após anuência da instituição e, aprovação pelo Comitê de Ética em Pesquisa (CEP), sob parecer $n^{\circ}$ 66861417.7.0000.5317. O estudo respeitou os preceitos éticos da Resolução $n^{\circ} 466$ de 2012, que discorre sobre as diretrizes e normas regulamentadoras de pesquisa envolvendo seres humanos (Brasil, 2012). Os participantes assinaram o Termo de Consentimento Livre e Esclarecido (TCLE), garantindo-lhes liberdade de participação e preservação da identidade. Para preservar o anonimato dos participantes, eles foram identificados pela letra "E" de ENFERMEIRO, seguida de um número em ordem crescente conforme entrevista, conforme exemplo: E1, E2, E3.

\section{Resultados e Discussão}

Ao iniciar a discussão dos resultados, torna-se relevante apresentar os sujeitos participantes; estes foram sete enfermeiros, cinco do sexo feminino; as idades variaram entre 26 e 47 anos; quanto ao tempo de atividade profissional no local do estudo houve uma variação de 14 a 144 meses; três dos participantes também eram enfermeiros assistenciais em outra instituição.

A partir da análise dos dados, foram elaboradas duas categorias temáticas: atuação do enfermeiro no atendimento ao paciente politraumatizado; e percepções dos enfermeiros sobre a prestação do serviço.

\section{Atuação do enfermeiro no atendimento ao paciente politraumatizado}

A administração do tempo na emergência é essencial para garantir uma qualidade nos resultados do atendimento e garantir suporte adequado à vida. A avaliação de possíveis lesões e a aplicabilidade de métodos de avaliação ao politraumatizado exige profissionais comprometidos com suas atribuições e dedicados a aprimorar suas habilidades, assim, além de preocuparem-se com os pacientes e com a assistência prestada, também se tornam uma referência para a equipe de trabalho.

Quando os enfermeiros foram questionados quanto ao seu papel frente ao atendimento do paciente politraumatizado, obteve as seguintes narrativas: 
E1: Bom, a minha atuação como enfermeira eu realizo procedimentos específicos do enfermeiro assim como direciono o atendimento as atividades do grupo técnico também, visando um atendimento rápido e eficiente visualizando o risco eminente de morte;

E3: Na minha visão o enfermeiro tem esse papel de liderança ele é o líder da equipe ele é o exemplo [...] comanda as ações que organiza a equipe de enfermagem.

Observou-se neste estudo que a prática da liderança em uma equipe torna a prestação de serviço mais eficaz e organiza o atendimento, uma vez que liderar cria uma conexão entre os profissionais. Deste modo, a equipe torna-se colaborativa, e qualifica o cuidado aos pacientes que necessitam de atendimento emergencial.

O enfermeiro possui importante atuação no atendimento ao paciente politraumatizado, possui capacidade técnica para ser um agente estratégico de promoção do trabalho em emergência, com habilidades de estimular, sensibilizar e articular a equipe para um trabalho humanizado. Logo, contribui para agilizar o atendimento à pessoa vítima de trauma, bem como outra situação de emergência (Miranda, Mendes, Oliveira, \& Santos, 2018; Santos, Lima, Pestana, Colomé, \& Erdmann, 2016).

Cabe ao enfermeiro, junto com a equipe, possuir aptidão teórica e prática, estar capacitado para realizar assistência qualificada considerando a individualidade de cada paciente, assim poderá oferecer o melhor suporte e aumentar a expectativa de vida diante do trauma (Neto, Costa, \& Mendonça, 2014).

Observa-se nos fragmentos que seguem uma preocupação em ter uma equipe que trabalhe em sincronia:

E3: [...] então quando a gente treina a equipe de enfermagem, quando a gente tem uma sala de emergência com uma equipe de enfermagem [...] já tem que saber suas posições, então não pode estar todo mundo correndo [...] a gente tem um papel de organização dessa equipe;

E6: [...] eu não vejo o atendimento do politraumatizado sem o enfermeiro atuando, não tem como porque é ele que faz todo o link com todo o resto, tem que agilizar a equipe de enfermagem.

Os enfermeiros do estudo se preocupavam com a qualidade do atendimento e trabalhavam motivados a fortalecerem os vínculos interdisciplinares para incentivar a troca de conhecimentos. Ao lançar esse olhar para liderança e gerenciamento, é necessário que se pense em outras habilidades e potencialidades no atendimento desse paciente, o que envolve a parte técnica, que está presente na prática diária desses profissionais. A seguir, os entrevistados se colocam a respeito de tais procedimentos e condutas:

E5: [...] então chega um paciente grave a primeira coisa que a gente vai fazer é ver se vai precisar de uma ventilação mecânica ou não [...] a gente segue na verdade o ABCDE [...] auxiliando o médico na parte da intubação;

E7: [...] a gente atenta pro nível de consciência, fazendo a escala de coma de Glasgow, a gente verifica sinais vitais, faz toda verificação do paciente para ver se não tem nenhum corto contuso, alguma fratura, se tem alguma fratura exposta ou só com deformidades, fizemos toda avaliação e cuidando sempre sinais vitais, encaminhamento para exames quando necessário, raio x de politrauma, tomografia, essa é a nossa atuação dos enfermeiros.

Este estudo demonstrou que os enfermeiros se colocavam como aqueles que devem estar sempre atentos a tudo que está acontecendo, seja com a equipe, na avaliação do paciente ou ainda no acompanhamento para realização de exames, eles se 
percebiam como intermediadores entre os membros da equipe.

O enfermeiro enquanto líder de equipe deve estabelecer práticas de organização nos mais diversos tipos de atendimento (Araszewski, Bolzan, Montezelli, \& Peres, 2014). Na enfermagem, a liderança torna-se um instrumento na construção da força de trabalho e transforma o ambiente, em um local saudável para trabalhar (Mattos, \& Balsanelli, 2019).

Pode-se perceber que, o gerenciamento é uma maneira de se complementar o processo de trabalho. De forma que, este profissional torna-se o articulador, responsável para estabelecer laços entre ações realizadas pelos profissionais da equipe, com finalidade de melhorar as práticas assistenciais, por mediação das relações estabelecidas no ambiente de trabalho. Assim, na emergência, o enfermeiro é considerado, o profissional que possibilita a promoção do trabalho em equipe (Santos, Lima, Pestana, Colomé, \& Erdmann, 2016).

Nesta conjuntura, o enfermeiro precisa ter conhecimento teórico-prático das condutas a serem tomadas na prática, isto inclui os procedimentos a serem realizados, a dinâmica do processo de enfermagem e a assistência qualificada, eficaz e segura (Will, Farias, Jesus, \& Rosa, 2020).

Além das competências citadas na avaliação inicial do paciente politraumatizado, os enfermeiros também se preocupavam em obter informações sobre a cinemática do trauma, a fim de identificar precocemente os recursos necessários, conforme a seguir:

E2: Como enfermeira, na chegada do paciente a gente avalia o paciente, vê se tem via aérea pérvia e se a coluna está imobilizada primeiramente, depois a gente conversa com o pré-hospitalar para saber qual foi a cinemática do trauma [...] expõe a vítima;

E4: Bom, a primeira coisa que eu me preocupo quando o paciente chega: o que que eu faço? Avaliar se ele está bem imobilizado, se ele não tem sangramento qual as queixas dele [...] referente se como e que foi a cinemática do trauma? Que tipo de acidente foi? [...] vou colocar ele na estabilização para avaliar ele por baixo da roupa quando não foi avaliado pelo SAMU.

Durante as falas identificou-se que para realizar uma avaliação completa do paciente politraumatizado, o profissional que realiza o atendimento deve estar apto e bem preparado, podendo avaliar através da cinemática as possíveis lesões que possa ter ocasionado a troca de energia entre os tecidos e o meio. Estas informações que são obtidas sobre a cena do acidente, propiciam buscas por pistas de possíveis lesões que estejam escondidas, fazendo com que sejam descobertas antes de trazerem complicações mais graves para a vítima.

Ainda, ao atender um politraumatizado, o enfermeiro deve estar sempre atento as lesões que o paciente apresenta, mas também aquelas que não estão visíveis no exame físico (National Association of Emergency Medical Technicians, 2019). Assim, conhecer a cinemática dele, possui importância na assistência a vítima, visto que, prever possíveis lesões permitirá a realização de uma abordagem mais direcionada, que poderá minimizar as sequelas do trauma e diminuir as possibilidades de morte (Sueoka, \& Abgussen, 2019).

Outros autores mencionam, a importância da presença do enfermeiro e de sua equipe no atendimento. $\mathrm{O}$ atendimento realizado em conjunto é indispensável desde as primeiras etapas de avaliação, consideram ainda que todos profissionais devem permanecer em sintonia, cada um realizando os procedimentos que lhes são cabíveis (Santos, Santos, Oliveira, \& Miranda, 2018).

Os enfermeiros destacaram que a equipe de enfermagem são os primeiros a entrarem em contato com a vítima, o que os permitem iniciar as primeiras avaliações, como mencionam: 
E1: O primeiro profissional que avalia é o enfermeiro e no momento que o paciente chega após a avaliação de enfermeiro é chamado o profissional médico;

E2: O primeiro profissional é o enfermeiro ou técnico de enfermagem, quando chega do pré-hospitalar é recebido pela equipe de enfermagem;

E6: Para mim é o enfermeiro [...] eu não fico esperando chamar o médico [...] eu acredito que o enfermeiro é o primeiro que tem que fazer essa avaliação.

Foi possível observar a preocupação na atuação do enfermeiro durante o atendimento. $\mathrm{O}$ fato de o enfermeiro ser o profissional que está presente desde o início do atendimento, acaba o tornando observador e coordenador, e para isso, deve estar sempre atualizado, cooperando com o serviço.

O enfermeiro quando está auxiliando o médico e realizando as primeiras etapas de atendimento, também está realizando a avaliação desse paciente. Entende-se assim que o enfermeiro é o profissional que liga múltiplas vertentes, assumindo seu papel de forma responsável prestando um atendimento de qualidade. Além disso, fica evidente que os enfermeiros possuem iniciativa e agilidade nas tomadas de decisão durante o atendimento.

$\mathrm{O}$ atendimento deve ser de forma rápida e eficiente, proporcionando um atendimento mais seguro para reduzir a mortalidade (Werlang, et al., 2017; Rodrigues, Santana, \& Galvão, 2017). Em concordância, o estudo de Lima destaca que, ao atender o paciente politraumatizado, com a sequência $\mathrm{ABCDE}$, o enfermeiro pode estar junto do médico atuando de forma a prevenir os agravos para o paciente e agilizar o processo de atendimento (Lima, 2014).

A sequência de $\mathrm{ABCDE}$ deve ser priorizada pelos profissionais na abordagem primária, através da abertura de vias aéreas com controle cervical (A); verificação de respiração (B); controle de grandes hemorragias com verificação de pulso (C); avaliação do nível de consciência (D), e exposição da vítima realizando o controle térmico (E). Já na abordagem secundária é realizado exame físico geral deste paciente e histórico deste paciente, iniciando pela observação detalhada da cabeça e identificação de lesões cortantes ou perfurantes, hematomas e crepitações (National Association of Emergency Medical Technicians, 2016).

\section{Percepções dos enfermeiros sobre a prestação do cuidado ao politraumatizado}

Dentro deste tema observou-se duas subcategorias: protocolos de atendimento ao paciente politraumatizado e; dificuldades e facilidades durante o atendimento de emergência.

\section{Protocolos de atendimento ao paciente politraumatizado}

Os enfermeiros destacaram que a instituição não dispõe de um protocolo de atendimento ao politraumatizado, porém, utilizam aqueles com validação internacional, como o Prehospital Trauma Life Support (PHTLS) e Advanced Trauma Life Support (ATLS), conforme os depoimentos a seguir:

E2: Aqui no Pronto Socorro a gente não tem um protocolo exclusivo, mas a gente usa o protocolo americano de trauma;

E3: A instituição ela tem como protocolo e como referência o PHTLS então a gente tem o ACLS que são protocolos internacionais, são protocolos americanos;

E6: Olha na realidade a gente usa o ABCDE dentro do APH e ATLS; 
E7: Então na instituição não tem protocolo, os profissionais quase todos têm o curso do APH, e a gente se baseia nesse protocolo né.

Em relação a periodicidade das atualizações, percebeu-se nas falas a seguir que os enfermeiros aproveitam a variedade de cursos disponíveis, mesmo não sendo disponibilizados pela instituição.

E3: [...] a gente faz uma revisão desse protocolo periodicamente [...] e a gente está sempre se atualizando, a instituição não nos dá esses cursos, mas a gente faz por conta [...] a gente também tem a obrigação de tá capacitando a equipe periodicamente;

E4: [...] faz um ano mais ou menos que eu fiz o APH e o BLS, de dois em dois anos renova, então, a gente tá sempre renovando, e o APH a gente tá sempre estudando através de livro, de cursos, até pela internet se pesquisando.

Em relação aos protocolos de atendimento, este estudo mostrou que a maioria dos enfermeiros se baseavam em protocolos internacionalmente reconhecidos, sobretudo não contavam com incentivo financeiro da instituição, tampouco educação permanente sobre a temática. Contudo, reconhecem a importância de se atualizarem quanto as mudanças de protocolos e disporem de recursos próprios para realizarem cursos.

No que tange ao serviço de urgência e emergência, educação permanente e treinamentos, o Ministério da Saúde alerta que treinamentos devem ser ofertados pelo serviço e ministrados de acordo com as atividades desenvolvidas (Brasil, 2014). A educação deve ser um instrumento permanente para estimular os trabalhadores as novas condutas para um melhor cuidado com a saúde (Lemos, 2016).

Desta forma, a possibilidade da oferta de educação permanente nos serviços de saúde, torna-se importante na vida do trabalhador, tendo em vista que prioriza aprendizagem no trabalho e pode proporcionar melhoria contínua na assistência (Tibola, et al., 2019). Neste contexto, em um estudo realizado com enfermeiros da unidade de emergência foi constatado que a instituição não oferece cursos de atualização. Ainda reforçam que o ideal seria a instituição planejar a promoção de melhores práticas, sendo custeado cursos de atualizações fora do hospital ou até mesmo desenvolvendo capacitações (Will, Farias, Jesus, \& Rosa, 2020).

\section{Facilidades e dificuldades durante o atendimento de emergência}

Esta temática surgiu da análise dos discursos dos enfermeiros em relação a questionamentos que pretendiam entender o que beneficiava ou interferia durante atendimento.

Pode-se observar nas falas que as dificuldades enfrentadas se baseiam na infraestrutura do serviço:

E1: [...] na sala da emergência, às vezes com a falta de bom senso da equipe médica que não se disponibiliza a reavaliação periódica, que a gente acaba aquele acúmulo de pacientes que daqui a pouco, reavaliados já poderiam ser liberados dali e por não ter também uma sala laranja, onde ficaria o paciente estabilizado aguardando, não sairia da área de risco, mas como a gente não disponibiliza o espaço físico fica todo mundo ali na sala de estabilização que atrapalha bastante;

E3: [...] a gente tem limitações estruturais a gente tem limitações de materiais em alguns momentos, então a gente tem uma realidade de interior [...] Ah eu acho que poderia ser melhor se a gente tivesse nível americano de 
politraumatizado né, o paciente chega, já ter avaliações que necessita de especialistas, exames, salas especificas de trauma, ter menos pacientes, mais enfermeiros, mais equipe pra trabalhar;

E4: [...] eu acho que a infraestrutura sim deveria sim ser um lugar diferenciado [...] então isso influencia bastante tu ter uma sala maior que tenha uma estrutura melhor, com certeza a gente consegue atender hoje mas poderia ser muito melhor, porque acontece as vezes de vim múltiplas vítimas.

Observa-se nas narrativas que a precária infraestrutura interfere no trabalho da equipe, pois frequentemente os pacientes ficam em observação na sala de estabilização, ambiente com uma área limitada, o que inviabiliza proporcionar conforto e não garante um serviço de qualidade.

A área física das unidades de urgência e emergência devem estar organizadas e estruturadas em conformidade com as necessidades da rede de atenção à saúde existente. Estas unidades devem dispor de infraestrutura física idealizada conforme tamanho, demanda, complexidade e perfil assistencial do serviço, com objetivo de garantir a segurança e continuidade da assistência ao paciente (National Association of Emergency Medical Technicians, 2016).

O objetivo destes serviços que atendem as emergências é proporcionar suporte rápido para os pacientes que necessitem de assistência. Para que isso seja possível, é necessário possuir uma boa infraestrutura, equipe qualificada, com facilidade na comunicação e agilidade na tomada de decisões, além de equipamentos e materiais de boa qualidade, a fim de proporcionar uma assistência integrada e humanizada, uma vez que, irá prestar atendimento a pacientes mais graves (Amestoy, et al., 2016; Silva, Bernardes, Gabriel, Rocha, \& Caldana, 2014).

Em relação às facilidades encontradas durante $o$ atendimento, dois enfermeiros citaram como facilidade o trabalho em equipe, como mostra os fragmentos a seguir:

E4: [...] todo pessoal que trabalha aqui tem um conhecimento diferente, tem uma experiência diferente né e trabalham porque gostam e a gente tá sempre ajudando uns aos outros e trocando essa experiência toda;

E5: [...] a equipe clínica e de enfermagem a gente trabalha tudo unido, eu acho que isso faz uma grande diferença, a gente trabalha meio que só com o olhar, cada um já sabe o que tem que fazer.

Constata-se nas falas que os profissionais vivenciavam uma constante troca de conhecimentos, o que pode contribuir para tornar o trabalho em equipe uma forma de agilizar os processos do cuidado e até mesmo problematizar, discutir e recuperar estratégias para viabilizar melhorias no serviço. Essas trocas são de suma importância, pois faz com que tenham melhor organização e desempenho, além de estimular a equipe a evoluir e aperfeiçoar os conhecimentos.

Os profissionais precisam trabalhar em sintonia na emergência, pois são fundamentais no processo de cuidar, sendo atribuído competências, habilidades, criatividade e sensibilidade. Falando criticamente, o cuidar em emergência demanda mais do que a parte técnica, ou seja, conta também com a experiência adquirida de seus profissionais (Amestoy, et al., 2016).

Neste sentido, a troca de conhecimentos entre os profissionais deve ser valorizada, como um método que acaba possibilitando novos rumos para qualificar o serviço. A Produção de ações que sejam criativas, pautadas em reciprocidade favorece o desenvolvimento de uma assistência efetiva (Amaral, Contim, Vieira, Chavaglia, \& Ohl, 2017).

Entende-se que o trabalho em equipe fortalece as relações interpessoais, na medida em que proporciona aumento do conhecimento e da autoconfiança em prol de uma assistência de qualidade dentro das possibilidades que a instituição oferece. Percebeu-se que as principais dificuldades dos enfermeiros estão relacionadas a estrutura física. A ausência de protocolos também foi mencionada, porém, os profissionais suprem esta carência com cursos de aperfeiçoamento. Evidenciou-se que os 
enfermeiros percebem a atuação de liderança como um diferencial desta categoria, com iniciativa durante a assistência e capacidade de coordenar ações gerenciais e assistenciais.

\section{Considerações Finais}

A partir dos resultados foi possível investigar a percepção dos enfermeiros sobre o atendimento ao paciente politraumatizado em um Pronto Socorro, conhecer sobre a participação deles durante o atendimento, os desafios vivenciados com infraestrutura e, as estratégias desempenhadas para suprir a falta de capacitação e educação permanente.

Identificou-se que o enfermeiro é o líder da equipe de enfermagem e seu trabalho se dá em equipe, existindo um elo com os demais profissionais da área da saúde, com o diferencial de participar ativamente no contexto multiprofissional ao fazer a integração entre médico, equipe de enfermagem e outros profissionais do serviço. Quanto aos procedimentos, percebeu-se que não há uma padronização, porém não destacam que isso cause prejuízo ao paciente.

Por fim, acredita-se que com todas as dificuldades enfrentadas pelos enfermeiros deste serviço ficou evidente o comprometimento, responsabilidade e o empenho pelo trabalho desenvolvido em prol do paciente politraumatizado.

Como limitação do estudo, observa-se que se a pesquisa tivesse um delineamento observacional possibilitaria verificar a atuação destes profissionais durante o atendimento ao politraumatizado, o que poderia dar maior confiabilidade aos achados. Os resultados obtidos indicam que esta temática precisa ser abordada em outras pesquisas, a fim de conscientizar os responsáveis pelas emergências de que o enfermeiro é um profissional essencial para um atendimento de qualidade ao politraumatizado, não apenas pela sua característica de liderança, mas sobretudo por ser um profissional que organiza e dinamiza o atendimento.

\section{Agradecimentos}

À Coordenação de Aperfeiçoamento de Pessoal de Nível Superior (CAPES) pela bolsa de doutorado da terceira autora.

\section{Referências}

Amaral, E. M. S., Contim, D., Vieira, D. S., Chavaglia, S. R. R., \& Ohl, R. I. B. (2017). Percepções sobre o trabalho da equipe de enfermagem em serviço hospitalar de emergência de adultos. Revista Mineira de Enfermagem; 8: 68- 87. http://www.dx.doi.org/10.5935/1415-2762.20170033

Amestoy, S. C., Lopes, R. F., Santos, B. P., Dornelles, C., Fuculo Junior, P. R. B., \& Santos, E. A. (2016). Exercício da liderança do enfermeiro em um serviço de urgência e emergência. Revista Eletrônica Gestão \& Saúde; 7(1): 38- 51. file:///C:/Users/User/Downloads/Dialnet-ExercicioDaLide rancaDoEnfermeiroEmUmServicoDeUrgen-5555878.pdf

Araszewski, D., Bolzan, M. B., Montezelli, J. H., \& Peres, A. M. (2014). O exercício da liderança sob ótica de enfermeiros de Pronto Socorro. Cogitare enferm; 19(1): 41- 47. http://dx.doi.org/10.5380/ce.v19i1.35933

Brasil. (2014). Portaria No 354, de 10 de março de 2014. Ministério da Saúde. http://bvsms.saude.gov.br/bvs/saudelegis/gm/2014/prt0354_10_03_2014.html

Brasil. Conselho Nacional de Saúde. (2012). Resolução n ${ }^{\circ}$ 466, de 12 de dezembro de 2012, no 12, Seção 1. https://bvsms.saude.gov.br/bv s/saudelegis/cns/2013/res0466_12_12_2012.html

Carvalho, I. C. C. M., \& Saraiva, I. S. (2015). Perfil das vítimas de trauma atendidos pelo serviço de atendimento móvel de urgência. Rev. Interd; 8(1): 137148. https://revistainterdisciplinar.uninovafapi.edu.br/index.php/revinter/article/view/392

Corrêa, A. R., Silva, B. P. A. R., \& Santiago, P. S. N. (2018). Atendimento pré-hospitalar: fatores facilitadores e dificultadores da assistência prestada por um grupo de regaste voluntário. Rev. enferm. Cent.-Oeste Min; 8: e2298. http://seer.ufsj.edu.br/index.php/recom/article/view/2298. https://docs.bvsalud.org/biblioref/2017/12/876137/4013-17985-1-pb.pdf

Lemos, C. L. S. (2016). Educação Permanente em Saúde no Brasil: educação ou gerenciamento permanente? Ciência \& Saúde Coletiva; 21 (3):913-922. https://dx.doi.org/10.1590/1413-81232015213.08182015

Lima, K. C. O. (2014). Cuidado do enfermeiro prestado ao indivíduo politraumatizado em unidade de emergência: uma revisão de literatura. (Trabalho de Conclusão de Curso). Universidade Federal de Santa Catarina, Santa Catarina, SC. 
Mattos, J. C. O., \& Balsanelli, A. P. (2019). A liderança do enfermeiro na atenção primária à saúde: revisão integrative. Enferm. Foco; 10 (4): 164-171. http://revista.cofen.gov.br/index.php/enfermagem/article/view/2618

Minayo, M. C. S. (2010). O desafio do conhecimento: pesquisa qualitativa em saúde. (12a ed.), Hucitec, p.407.

Miranda, V. M., Mendes, J. R., Oliveira, A. D. S., \& Santos, A. M. R. (2018). Assistência do enfermeiro ao politraumatizado: revisão integrativa. Rev. Enferm. UFPI [internet]; 7(2):79-85. http://www.ojs.ufpi.br/index.php/reufpi/article/view/6593/pdf.

National Association of Emergency Medical Technicians. (2016). Prehospital Trauma Life Support (PHTLS) atendimento pré-hospitalar ao traumatizado. Porto Alegre, RS: Grupoa.

National Association of Emergency Medical Technicians. (2019). PAC: PHTLS 9E: PREHOSP Trauma course manual w/nav ebook. Artmed.

Neto, V. L. S., Costa, M. A. D. J., \& Mendonça, A. E. O. (2014). Abordagem propeudêuticas de enfermagem ao paciente com trauma raquimedular no pronto atendimento. Rev. Univ. Vale Rio Verde 12(2): 716-724. http://dx.doi.org/10.5892/ruvrd.v12i2.1529

Pereira A. S., Shitsuka D. M., Parreira, F. J., \& Shitsuka, R. (2018). Metodologia da pesquisa científica. UFSM. https://repositorio.ufsm.br/bitstream/h andle/1/15824/Lic_Computacao_Metodologia-Pesquisa-Cientifica.pdf?sequence=1

Praça, W. R., Matos, M. C. B., Magro, M. C. S., Fioravanti, R. K., \& Hermann, P. R. S. (2017). Perfil epidemiológico e clínico de vítimas de trauma em um hospital do Distrito Federal. Rev. Prev. Infec. Saúde; 3(1):1-7. https://revistas.ufpi.br/index.php/nupcis/article/view/6219

Rodrigues, M. S., Santana, L. F., \& Galvão, I. M. (2017). Utilização do ABCDE no atendimento do traumatizado. Rev. med. (São Paulo); 96(4):278-80. http://dx.doi.org/10.11606/issn.1679-9836.v96i4p278-280

Santos, J. L. G., Lima, M. A. D. S., Pestana, A. L., Colomé, I. C. S., \& Erdmann, A. L. (2016). Estratégias utilizadas pelos enfermeiros para promover o trabalho em equipe em um serviço de emergência. Rev. gaúch. Enferm; 37(1):e50178. http://dx.doi.org/10.1590/1983-1447.2016.01.50178

Santos, M. A. S. S., Santos, L. G. E., Oliveira, G. F. S. M., \& Miranda, L. N. (2018). Assistência de enfermagem ao paciente politraumatizado. Ciências Biológicas e de Saúde Unit; 4(2):11-22. https://periodicos.set.edu.br/fitsbiosaude/article/view/4648/2777

Silva, D. S., Bernardes, A., Gabriel, C. S., Rocha, F. L. R., \& Caldana, G. (2014). A liderança do enfermeiro no contexto dos serviços de urgência e emergência. Rev. Eletr. Enf. [Internet]; 16(1):211-9. http://dx.doi.org/10.5216/ree.v16i1.19615.

Sontag R. (2014). A estruturação da Rede de Urgência e Emergência na "13a Região da diversidade": Um relato de experiência [Monografia] Departamento de Enfermagem: Universidade Federal de Santa Catarina. https://repositorio.ufsc.br/xmlui/handle/123456789/173506

Sueoka, J. S, \& Abgussen, C. M. B. (2019). Cinemática do Trauma. APH resgate: emergência em trauma. Elsevier.

Tibola, T. S. A., Cordeiro, A. L. P., Stacciarin, T. S. G., Engel, R. H., Costa, D. G., \& Haas, V. J. (2019). Fatores que influenciam a participação dos profissionais de enfermagem na educação permanente em hospital público. Enferm Foco [Internet]; 10(2): 11-16. http://revista.cofen.gov.br/index.php/enfermagem/article/view/2044/532

Werlang, S. L., Badke, M. R., Freitag, V. L., Silva, G. S., Federizzi, D. S., \& Ribeiro, M. V. (2017). Enfermagem na Assistência ao Traumatismo Cranioencefálico em um Hospital Universitário. J Health Sci; 19(2):177-82. https://docs.bvsalud.org/biblioref/2017/12/876137/4013-17985-1-pb.pdf

Will, R. C., Farias, R. G., Jesus, H. P., \& Rosa, T. (2020). Cuidados de enfermagem aos pacientes politraumatizados atendidos na emergência. Nursing 23(262): 3766-3777. http://www.revistanursing.com.br/revistas/263/pg 109.pdf

Zappas, Z., Costa, A. E. K da, \& Pissaia, L. F. (2018). Qualidade de vida em enfermeiros atuantes em uma instituição hospitalar. Revista Espaço Ciência \& Saúde; 6(3): 3-13. http://revistaeletronicaocs.unicruz.edu.br/index.php/enfermagem/article/view/6757 